\title{
Kaposi's Varicelliform-Like Eruption in a Patient Treated with Everolimus for Metastatic Renal Cell Carcinoma: Report of a Rare Case
}

\author{
Soojung Hong ${ }^{a, b}$ Eun Hye Kim ${ }^{b}$ Sung Bin Cho ${ }^{c}$ Sun Young Rha ${ }^{b, d, e}$ \\ ${ }^{a}$ Department of Internal Medicine, National Health Insurance Service Ilsan Hospital, \\ Goyang, Departments of ${ }^{b}$ Internal Medicine and 'Dermatology, and ${ }^{\mathrm{d}}$ Institute for Cancer \\ Research, Yonsei University College of Medicine, and ${ }^{\mathrm{e}}$ Brain Korea 21 Project for Medical \\ Science, Seoul, Korea
}

\section{Key Words}

Kaposi's varicelliform eruption - Renal cell carcinoma $\cdot$ Mammalian target of rapamycin inhibitor

\begin{abstract}
Kaposi's varicelliform eruption is a cutaneous eruption caused by the herpes simplex virus and a few other viruses that infect persons with pre-existing dermatosis such as atopic dermatitis. We report the case of a 56-year-old man who was treated with the mammalian target of rapamycin inhibitor, everolimus, for metastatic renal cell carcinoma. He presented with painful, umbilicated vesicles and pustules on his face, genital region, forearms, and legs suggestive of Kaposi's varicelliform eruption. He did not have a history of any visceral viral disease and pre-existing dermatosis. The diagnosis was based on the clinical features. He was treated with acyclovir for 7 days, with improvement of his skin lesions. We discuss the clinical manifestations of the Kaposi varicelliform-like eruption in an immunocompromised patient treated with everolimus.

(c) 2014 S. Karger AG, Basel
\end{abstract}

\section{Introduction}

Kaposi's varicelliform eruption is a disseminated cutaneous infection usually caused by the herpes simplex virus (HSV). It predominantly occurs in patients with pre-existing active dermatosis [1] and is characterized by disseminated vesiculopustules and erosions. It is 
common in patients with atopic dermatitis and rarely develops in an immunocompromised host. There have been several case reports of Kaposi's varicelliform eruption occurring in patients with cutaneous T-cell lymphoma and multiple myeloma [2, 3]. Herein, we report a nonfatal case of Kaposi's varicelliform-like eruption occurring in an immunocompromised patient taking the mammalian target of rapamycin (mTOR) inhibitor, everolimus, for metastatic renal cell carcinoma (RCC).

\section{Case Report}

A 56-year-old man presented with a 6-day history of fever, chills, and severe forehead pain and 5 days of vesicular eruptions involving the skin of the forehead, both eyelids, and nose. The skin lesions spread to his chest, back, and bilateral upper and lower extremities over the subsequent 4 days (fig. 1a, b). The patient had not had chickenpox during childhood or any recent exposure to it.

He had been diagnosed with left RCC in June 2007. He underwent radical nephrectomy and the pathologic type was clear cell carcinoma with T3N0M0, stage III, Fuhrman grade II. The patient was given sorafenib $400 \mathrm{mg}$ twice daily for 7 months as adjuvant treatment. Follow-up computed tomography of the abdomen and pelvis performed 8 months after surgery revealed recurrence of the lesion in the left renal fossa invading the tail of the pancreas, transverse colon, and spleen. He underwent splenectomy, distal pancreatectomy, and segmental resection of the transverse colon. Two months later, computed tomography showed three metastatic nodules in the liver, and the patient underwent liver metastasectomy. After surgery, he was started on $37.5 \mathrm{mg}$ of sunitinib, which he took for 7 months. Eleven months after the liver surgery, disease progression involving the liver and lungs was detected, and the patient was enrolled in a clinical trial of second-line treatment using RAD001 (everolimus), which he took for 19 months. Thereafter, disease progressed in his lungs and the patient took palliative sunitinib on a 4-week-on and 2-week-off schedule. Seven months later, a whole-body bone scan revealed multiple bone metastases involving the left ribs, left proximal femur, left ischium, and right iliac bone. The patient underwent palliative radiation therapy (45 Gy) for the pain-causing left ribs and weight bearing both pelvic bones. After finishing the radiation therapy, the patient began everolimus as palliative treatment. He took everolimus for 10 days before the appearance of the rash. The patient had stopped the drug 3 days before admission.

At admission, the patient's temperature was $38.1^{\circ} \mathrm{C}$, his blood pressure was $151 / 102$ $\mathrm{mm} \mathrm{Hg}$, and the pulse rate was 110 beats/min. He had vesicles and pustules, with crusting and swelling on the skin of his forehead, both eyelids and nose. Both eyelids were also tender and red. Vesicles, pustules and scabs were present on his trunk and extremities as well. He also had grade 2 oral mucositis. No lymphadenopathy was present.

The patient's complete blood count revealed a white blood count of $4,640 / \mu$ l (neutrophils $2,210 / \mu \mathrm{l}$, lymphocytes $1,370 / \mu \mathrm{l}$ ), hemoglobin of $13.0 \mathrm{~g} / \mathrm{dl}$, and platelet count of $79,000 / \mu \mathrm{l}$ presenting mild lymphopenia and thrombocytopenia. The C-reactive protein (CRP) was elevated at $85.8 \mathrm{mg} / \mathrm{l}$ (normal range, $0-8$ ). The results of other laboratory studies, including routine biochemistry and chest X-rays, were normal. The CD4 and CD8 lymphocyte counts were $0.28 \times 10^{9} / \mathrm{l}$ and $0.64 \times 10^{9} / \mathrm{l}$, respectively $(\mathrm{CD} 4: \mathrm{CD} 8$ ratio $=0.44)$. Serology for human immunodeficiency virus was negative. His serum IgM and IgG antibodies were negative for varicella-zoster virus. HSV type 1 and type 2 were also undetectable by polymerase chain reaction assay. 
Hong et al:: Kaposi's Varicelliform-Like Eruption in a Patient Treated with Everolimus for Metastatic Renal Cell Carcinoma: Report of a Rare Case

A dermatologic clinical diagnosis of Kaposi's varicelliform eruption was made based on the patient's typical skin manifestations, including viral infection-like blisters and eruptions. The patient was intravenously administered $250 \mathrm{mg}$ of acyclovir every $8 \mathrm{~h}$. Twenty-four hours after initiation of treatment, there were no new vesicles, and the patient's clinical status improved. However, his fever did not completely resolve. We reconsulted the dermatologist, and skin biopsy was performed after 6 days of acyclovir.

The biopsy showed interstitial granulomatous dermatitis with a few eosinophils, extravasated red blood cells and basal vacuolization (fig. 2). There was no evidence of herpetic viral infection in the biopsy specimen. The pathologic findings were suggestive of a granulomatous drug eruption. Therefore, we concluded that the patient had an everolimusinduced drug eruption manifesting as Kaposi's varicelliform eruption considering both clinical presentation and pathologic findings.

After stopping everolimus, a follow-up bone scan of the patient revealed new spinal metastases involving the T3 and L1 vertebrae and a compression fracture of L3. He had palliative radiation therapy ( $42 \mathrm{~Gy}$ ) of his thoracic and lumbar spine. Three months later, the patient died of disease progression of the metastatic RCC.

\section{Discussion}

Kaposi's varicelliform eruption is characterized by dissemination of cutaneous infection of HSV, and some other viruses in patients with pre-existing dermatoses [2, 4]. It is also called eczema herpeticum because it is most commonly seen in patients with atopic dermatitis [5, 6]. Kaposi's varicelliform eruption has also been described in association not only with dermatologic conditions, such as psoriasis, lupus vulgaris, contact dermatitis, and rosacea, but also malignant diseases such as cutaneous T-cell lymphoma and multiple myeloma $[2,3,7]$. There has been a single report of a patient with Kaposi's varicelliform eruption associated with a drug reaction (phenytoin) [1].

The severity of Kaposi's varicelliform eruption appears to be unrelated to the extent of the eczematous lesions, and active dermatitis is not necessary for the development of eruption. Systemic viremia involving multiple organs, including the liver, lungs, brain, gastrointestinal tract, adrenal glands, and eyes, is the major cause of morbidity and mortality [8]. Transmission can occur through contact with infected persons or by dissemination of primary or recurrent herpes. Recurrent episodes may also occur because the virus persists in the host and periodically reactivates in the mucosa and skin; however, the manifestations of recurrent episodes are milder and usually not systemic [8].

The diagnosis of Kaposi's varicelliform eruption is mainly clinical. There are several tests that can be useful. A Tzanck smear can provide rapid diagnosis when it shows the characteristic epithelial multinucleated giant cells. Polymerase chain reaction assays can be used to detect a virus. Both biopsy and serology are of little diagnostic value, and these are not recommended as routine tests [5, 9].

Our patient did not have pre-existing dermatitis, but was taking everolimus for the treatment of the metastatic RCC. As an mTOR inhibitor, everolimus modulates T-lymphocyte homeostasis [10], and is therefore used for immunosuppression after organ transplantation and as an anticancer therapeutic. The patient first took everolimus for 19 months in a clinical trial and achieved stable disease. At that time, he only had grade 1 mucositis, insomnia, and hypertriglyceridemia but no skin manifestations of toxicity. However, when taking everolimus for a second time, he developed a drug rash and pruritus after 10 days on everolimus, which were followed by clusters of umbilicated vesiculopustules. These 
progressed to painful hemorrhagic, crusted, and punched-out erosions. Administration of everolimus was stopped, and the patient started with the antiviral therapy. His condition improved after administration of acyclovir.

The exact factors for Kaposi's varicelliform eruption are still unclear. However, many studies commonly explained two factors. A defective epidermal barrier and immunosuppression associated with either therapy or a debilitated state of the patient may lead to disseminated HSV infection [1]. In a retrospective review of 100 atopic dermatitis patients with Kaposi's varicelliform eruption, a high IgE serum level was found to be a risk factor [5]. Defective cytokine secretion and decreased cell-mediated immunity is important in the control of primary and recurrent HSV infections [6]. The suppressed immunity and the loss of the epidermal barrier function as a result of everolimus may have contributed to the development of Kaposi's varicelliform-like eruption in our patient, who had an increasing tumor burden and a worsening performance status.

Some investigators have shown that markers of tumor inflammation or host immune status, such as CRP and neutrophil-to-lymphocyte ratio, may be predictive to survival outcome $[10,11]$. At admission, the patient had a high CRP level, mild lymphopenia, and a relatively high neutrophil-to-lymphocyte ratio, which taken together, reflected the poor condition of the patient.

We ultimately diagnosed the patient with Kaposi's varicelliform-like eruption associated with a drug eruption based on the pathologic findings, clinical manifestations, and the clinical course of the patient after everolimus treatment. Unfortunately, we were unable to confirm a viral infection, perhaps because of delays in performing the skin biopsy and other laboratory tests. However, we believe that the most important tool for the diagnosis of Kaposi's varicelliform eruption is clinical judgment.

This is a rare case of Kaposi's varicelliform-like eruption occurring as an everolimusinduced rash. Physicians need to be aware that patients with increasing tumor activity and decreasing immunity may be at risk for this problem. Early diagnosis, stopping the suspected medication and appropriate treatment of patients at risk for viral complications are very important medical considerations.

\section{Disclosure Statement}

The authors have no conflicts of interest to disclose.

\section{References}

1 Ajith C, Dogra S, Handa S: Kaposi's varicelliform eruption in a patient with phenytoin-induced drug rash. Int J Dermatol 2006;45:1452-1453.

-2 Fukuzawa M, Oguchi S, Saida T: Kaposi's varicelliform eruption of an elderly patient with multiple myeloma. J Am Acad Dermatol 2000;42:921-922.

-3 Masessa JM, Grossman ME, Knobler EH, Bank DE: Kaposi's varicelliform eruption in cutaneous T cell lymphoma. J Am Acad Dermatol 1989;21:133-135.

-4 Smith BD, Son CB, Wilson LD: Disseminated herpes simplex after total skin electron beam radiotherapy for mycosis fungoides. J R Soc Med 2003;96:500-501.

5 Olson J, Robles DT, Kirby P, Colven R: Kaposi varicelliform eruption (eczema herpeticum). Dermatol Online J 2008;14:18.

6 Peng WM, Jenneck C, Bussmann C, et al: Risk factors of atopic dermatitis patients for eczema herpeticum. J Invest Dermatol 2007;127:1261-1263.

-7 Paradisi A, Capizzi R, Guerriero G, Rotoli M, Bussoletti C, Amerio PL: Kaposi's varicelliform eruption complicating allergic contact dermatitis. J Am Acad Dermatol 2006;54:732-733.

8 Shenoy MM, Suchitra U: Kaposi's varicelliform eruption. Indian J Dermatol Venereol Leprol 2007;73:65. 


\section{Case Reports in Oncology}

\begin{tabular}{l|l}
\hline Case Rep Oncol 2014;7:337-342 & \\
\hline DOI: $10.1159 / 000362925$ & $\begin{array}{l}\text { ○ 2014 S. Karger AG, Basel } \\
\text { www.karger.com/cro }\end{array}$ \\
\hline
\end{tabular}

Hong et al.: Kaposi's Varicelliform-Like Eruption in a Patient Treated with Everolimus for Metastatic Renal Cell Carcinoma: Report of a Rare Case

9 Hayashi S, Yamada Y, Dekio S, Jidoi J: Kaposi's varicelliform eruption in a patient with mycosis fungoides. Clin Exp Dermatol 1997;22:41-43.

10 Thiery-Vuillemin A, Laheurte C, Mansi L, et al: Immunomodulatory effects of everolimus in a long responsive patient with metastatic renal cell carcinoma. J Immunother 2014;37:51-54.

11 Santoni M, De Giorgi U, Iacovelli R, et al: Pre-treatment neutrophil-to-lymphocyte ratio may be associated with the outcome in patients treated with everolimus for metastatic renal cell carcinoma. Br J Cancer 2013;109:1755-1759.

S. Hong and E.H. Kim contributed equally to this work.
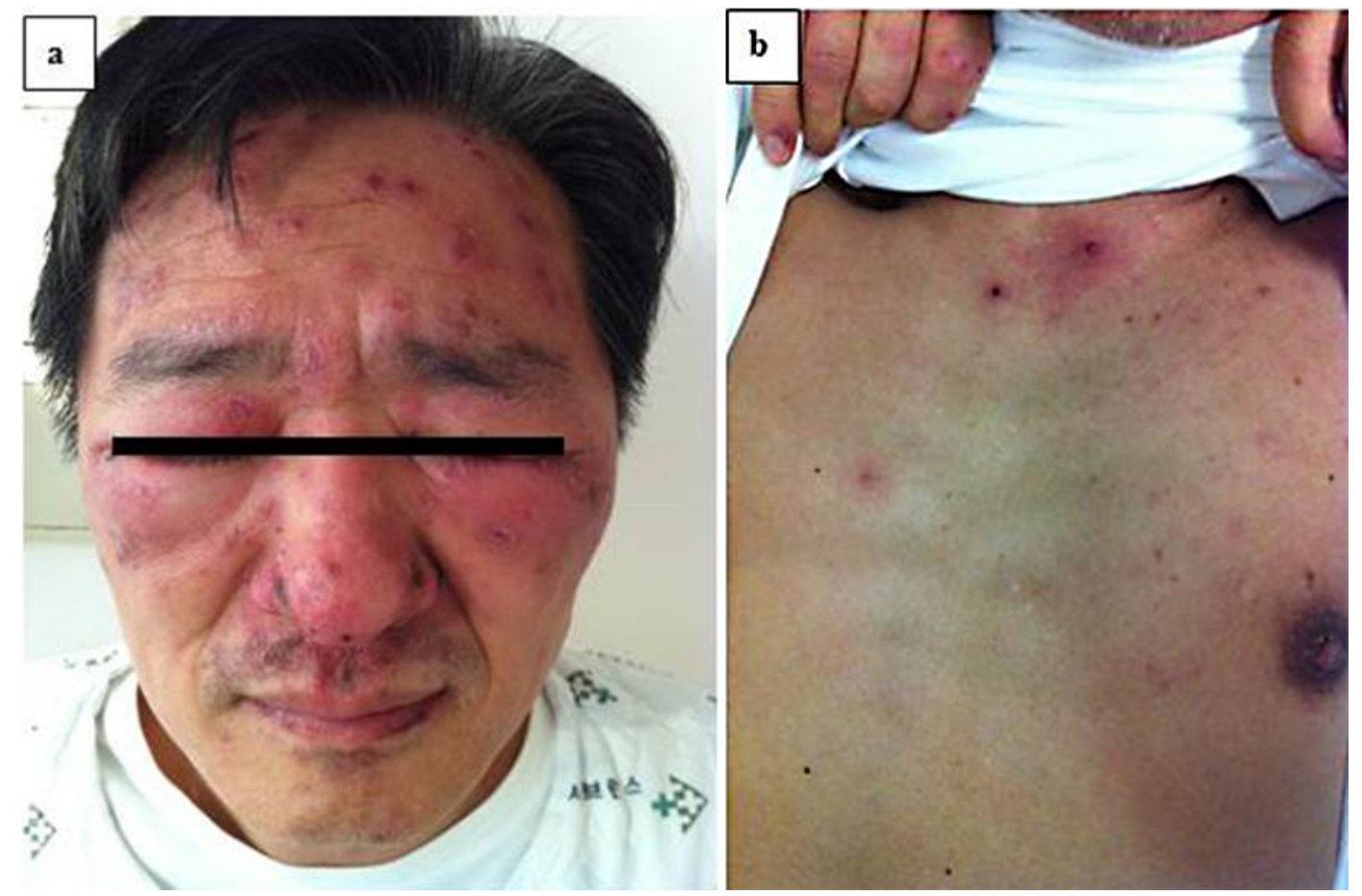

Fig. 1. Clusters of erythematous papules and crusts are seen on the patient's face (a). Erythematous papules and crusts are scattered on the other areas of the trunk (b). 


\section{Case Reports in Oncology}

Case Rep Oncol 2014;7:337-342

DOI: $10.1159 / 000362925$

2014 S. Karger AG, Basel

www.karger.com/cro

Hong et al.: Kaposi's Varicelliform-Like Eruption in a Patient Treated with Everolimus for Metastatic Renal Cell Carcinoma: Report of a Rare Case

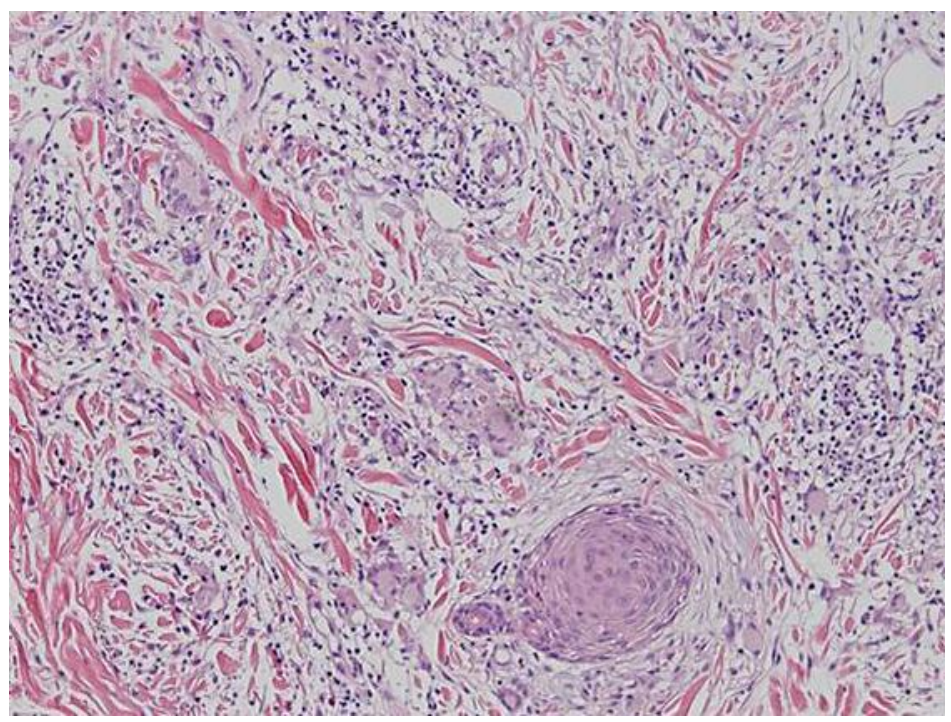

Fig. 2. Skin biopsy showing interstitial granulomatous dermatitis with a few eosinophils, extravasated red blood cells and basal vacuolization. 